\title{
Erratum to: Novel pH-Sensitive Microbial Rhodopsin from Sphingomonas paucimobilis
}

\author{
N. Maliar ${ }^{a}$, I. S. Okhrimenko ${ }^{a}$, L. E. Petrovskaya ${ }^{b}$, A. A. Alekseev ${ }^{a, d, e, f}$, K. V. Kovalev ${ }^{a, c, d, e, f}$, \\ D. V. Soloviov ${ }^{a, g, h}$, P. A. Popov ${ }^{i, a}$, T. I. Rokitskaya ${ }^{j}$, Y. N. Antonenko ${ }^{\prime}$, D. V. Zabelskii ${ }^{a, d, e}$, \\ D. A. Dolgikh ${ }^{b, k}$, Academician M. P. Kirpichnikov ${ }^{b, k}$, and V. I. Gordeliy ${ }^{a, c, d, e, *}$ \\ Received January 28, 2021; revised January 28, 2021; accepted January 28, 2021
}

DOI: $10.1134 / \mathrm{S} 1607672921020198$

Page 345, Fig. 3a, caption (a) should read as follows:

(a) Change in $\mathrm{pH}$ of a suspension of E. coli cells expressing SpaR under illumination with and without the addition of CCCP (100 mM NaCl, pH 6.2).

Page 345, Fig. 3b, the following sentence should be added to caption (b):

(a)

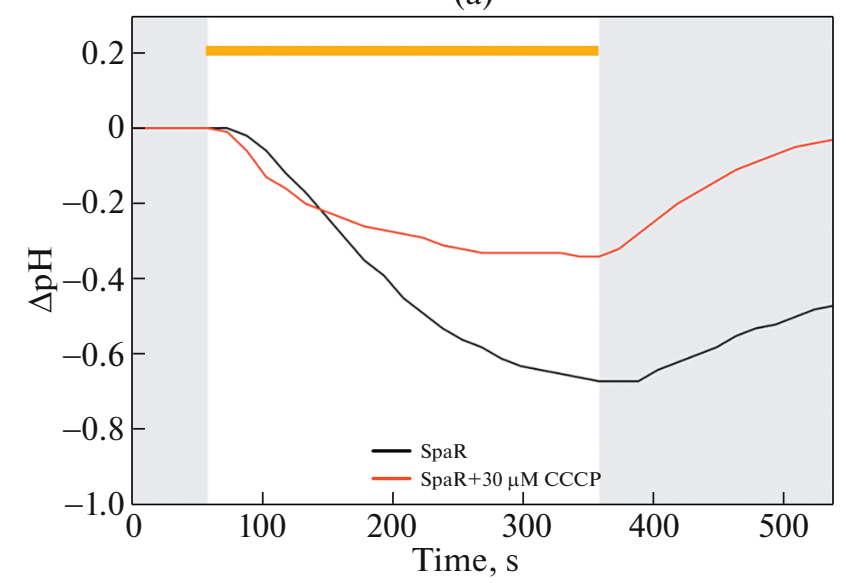

(b)

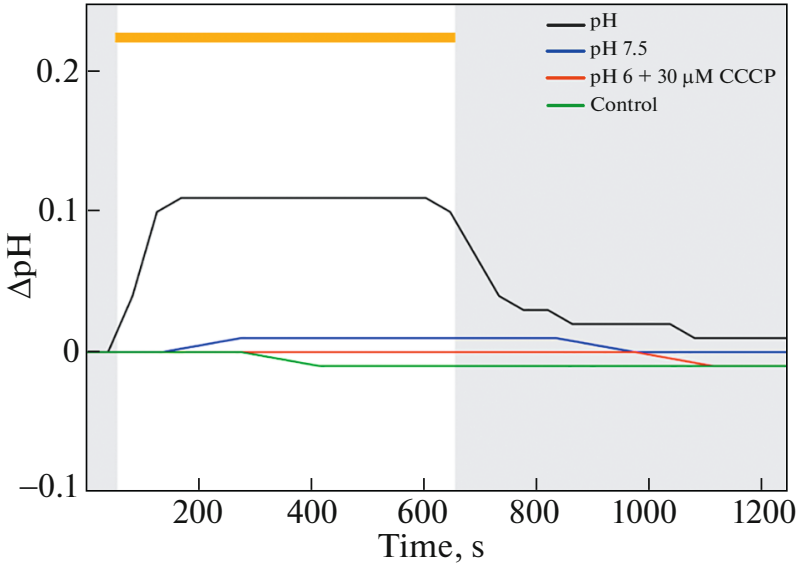

Black line refers to the measurements at $\mathrm{pH} 6$.

The original article can be found online at https://doi.org/10.1134/S1607672920060162

\footnotetext{
${ }^{a}$ Moscow Institute of Physics and Technology, Dolgoprudnyi, Moscow oblast, Russia

${ }^{b}$ Shemyakin and Ovchinnikov Institute of Bioorganic Chemistry, Russian Academy of Sciences, Moscow, Russia

${ }^{c}$ Institut de Biologie Structurale (IBS), Universitŭ Grenoble Alpes, CEA, CNRS, Grenoble, France

${ }^{d}$ Institute of Biological Information Processing (IBI-7: Structural Biochemistry), Forschungszentrum Jülich GmbH, Jülich, Germany

e JuStruct: Jülich Center for Structural Biology, Forschungszentrum Jülich GmbH, Jülich, Germany

${ }^{f}$ Institute of Crystallography, University of Aachen (RWTH), Aachen, Germany

${ }^{g}$ Institute for Safety Problems of Nuclear Power Plants, National Academy of Sciences of Ukraine, Kyiv, Ukraine

${ }^{h}$ Joint Institute for Nuclear Research, Dubna, Russia

${ }^{i}$ Skolkovo Institute of Science and Technology, Moscow, Russia

${ }^{j}$ Belozersky Institute of Physico-Chemical Biology, Moscow State University, Moscow, Russia

${ }^{k}$ Biological Faculty, Moscow State University, Moscow, Russia

*e-mail: valentin.gordeliy@ibs.fr
} 